André Luiz Mendoza

\title{
Gestão da Cadeia de Suprimentos Global na Glaxosmithkline - GSK
}

Dissertação apresentada como requisito parcial para obtenção do título de Mestre em Logística pelo Programa de Pós-Graduação em Engenharia Industrial da PUC-Rio.

Orientador: Luiz Felipe R. R. Scavarda do Carmo

Rio de Janeiro

Novembro de 2006 


\title{
Gestão da Cadeia de Suprimentos Global na Glaxosmithkline - GSK
}

\begin{abstract}
Dissertação apresentada como requisito parcial para obtenção do título de Mestre (opção profissional) pelo Programa de Pós-Graduação em Engenharia Industrial da PUC-Rio. Aprovada pela Comissão Examinadora abaixo assinada.
\end{abstract}

Prof. Luiz Felipe R. R. Scavarda do Carmo Orientador Departamento de Engenharia Industrial - PUC-Rio

Prof. José Eugênio Leal Departamento de Engenharia Industrial - PUC-Rio

Prof. Silvio Hamacher Departamento de Engenharia Industrial - PUC-Rio

Prof. José Eugênio Leal Coordenador(a) Setorial do Centro Técnico Científico - PUC-Rio 
Todos os direitos reservados. É proibida a reprodução total ou parcial do trabalho sem autorização da universidade, do autor e do orientador.

\section{André Luiz Mendoza}

Graduou-se em Comunicação social na FACHA (Faculdades Integradas Helio Alonso) em 1997. Trabalha na Glaxosmithkline desde 1993 ingressando na área de marketing e vendas. Iniciou a carreira em logística como gerente de demanda no ano 2000, passando logo depois pela área internacional onde ajudou a implementar diversos projetos em vários países. Atualmente exerce a função de gerente de distribuição para a região internacional baseado na sede da companhia em Londres.

Ficha Catalográfica

Mendoza, André Luiz

Gestão da cadeia de suprimentos global na Glaxosmithkline - GSK / André Luiz Mendonza ; orientador: Liz Felipe R. R. Scavarda do Carmo. - 2006.

$95 \mathrm{f} . ; 30 \mathrm{~cm}$

Dissertação (Mestrado em Engenharia Industrial)Pontifícia Universidade Católica do Rio de Janeiro, Rio de Janeiro, 2006.

Inclui bibliografia

1. Engenharia industrial - Teses. 2. Gestão da Cadeia de Suprimentos. 3. Gerenciamento dos Suprimentos e Demanda. 4. Indústria Farmacêutica. I. Carmo, Luiz Felipe R. R. Scavarda do. II. Pontifícia Universidade Católica do Rio de Janeiro. Departamento de Engenharia Industrial. III. Título.

CDD: 658.5 


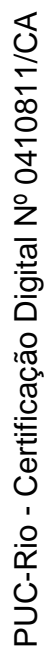

Para o meu Pai, que nos deixou para descansar em paz uma semana após a defesa desta dissertação. 


\section{Agradecimentos}

A minha esposa Verônica como fonte de incentivo e amor. Principalmente por ter abdicado de muita coisa para me suportar neste e em outros projetos.

Aos meus filhos Maria e Rodrigo que mudaram minha perspectiva de vida.

Ao meu Orientador e amigo Professor Luiz Felipe Scavarda um agradecimento especial pela tolerância, persistência, confiança e ajuda inestimável na conclusão desta dissertação.

Aos meus pais, Wilson e Creusa e irmão Eduardo como fonte de apoio indeterminado.

Aos componentes da comissão examinadora professores José Eugênio Leal e Silvio Hamacher pela oportunidade de defender esta dissertação em condições tão excepcionais.

Aos meus amigos da turma do mestrado pelos momentos fabulosos que passamos juntos. 


\section{Resumo}

Mendoza, André Luiz; Scavarda do Carmo, Luiz Felipe R. R.. Gestão da Cadeia de Suprimentos Global na Glaxosmithkline - GSK. Rio de Janeiro, 2006. 95p. Dissertação de Mestrado - Departamento de Engenharia Industrial, Pontifícia Universidade Católica do Rio de Janeiro.

As companhias farmacêuticas necessitam dirigir atenção à demanda crescente em trazer medicamentos ao mercado em uma forma econômica. A pressão sobre o preço está aumentando, e as vantagens de ter um período de exclusividade da utilização de suas patentes estão diminuindo, enquanto a produção de produtos genéricos está crescendo em todo mundo. Ter um portifólio de novas drogas é crítico, mas também é crítico ter habilidade de trazer essas drogas ao mercado em uma maneira eficiente e de baixo custo. Entretanto, as companhias farmacêuticas não podem mais contar unicamente em pesquisa e desenvolvimento e marketing para sobreviver, mas devem também confiar nos valores agregados pela gestão da cadeia de suprimentos (Supply Chain Management - SCM). Neste contexto, o presente trabalho tem o intuito de analisar o modelo de SCM global utilizado por uma grande empresa de medicamentos (Glaxosmithkline-GSK), através da apresentação das principais características de sua cadeia de suprimentos, da análise do planejamento e gerenciamento do suprimento e da demanda e da análise de práticas de SCM implementadas por esta empresa na Europa.

\section{Palavras-chave:}

Gestão da Cadeia de Suprimentos; Gerenciamento dos Suprimentos e Demanda; Indústria Farmacêutica. 


\section{Abstract}

Mendoza, André Luiz; Scavarda do Carmo, Luiz Felipe R. R. Global Supply Chain Management at GlaxoSmithKline - GSK. Rio de Janeiro, 2006. 95p. M.Sc. Dissertation - Departamento de Engenharia Industrial, Pontifícia Universidade Católica do Rio de Janeiro.

Pharmaceutical companies need to address the growing demand for bringing medicines to market in an economical way. Price pressure is increasing, and the advantages of having a buffered patent window is decreasing as generic manufacturing is on the rise. Having a pipeline of new drugs is critical, but it is also critical the ability to bring them to market in a cost effective and efficient manner. However, pharmaceutical companies can no longer solely rely on research and development and marketing to survive, but must also rely on supply chain management to deliver value. Within this context, this dissertation intends to analyze the supply chain management model of a major pharmaceutical company (GlaxoSmithkline - GSK) throughout an overview of the key characteristics of its supply chain, supply and demand management and some examples of projects implemented in Europe.

\section{Keywords:}

Supply Chain Management; Supply and Demand Management; Pharmaceutical Industry. 
LISTA DE FIGURAS

LISTA DE TABELAS

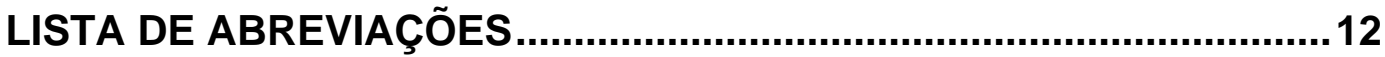

1 INTRODUÇÃO

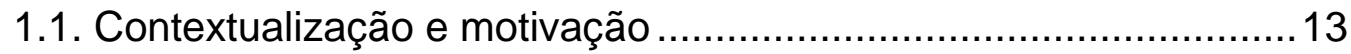

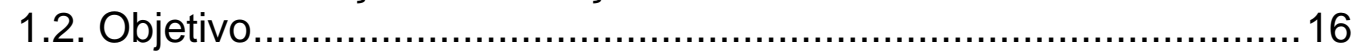

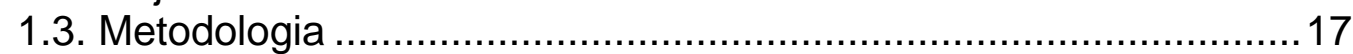

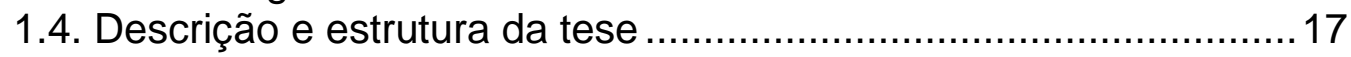

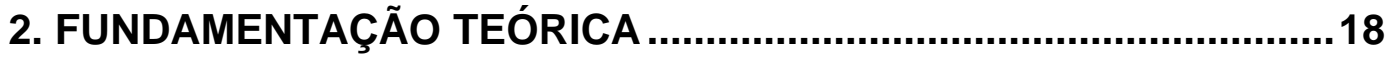

2.1. Sistemas de Gestão da Demanda e de Suprimentos ....................18

2.1.1. MRP (Material Requirement Planning) ……………................. 19

2.1.2. MRPII (Manufacturing Resource Planning).................................22

2.1.3. S\&OP (Sales and Operation Planning) ......................................... 24

2.1.4. DRP (Distribution Resource Planning) ......................................26

2.1.5. ERP (Enterprise Resource Planning) ....................................2

2.2. Iniciativas colaborativas ...........................................................29

2.2.1. CPFR (Collaborative Forecasting Planning and Replenishment) 30

2.2.2. VMI (Vendor Managed Inventory) .............................................33

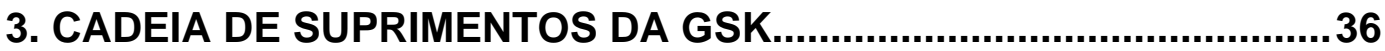

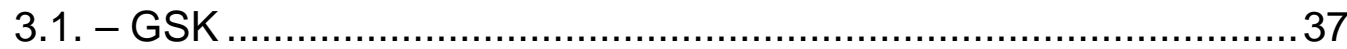

3.2. A cadeia de suprimentos ………........................................... 38

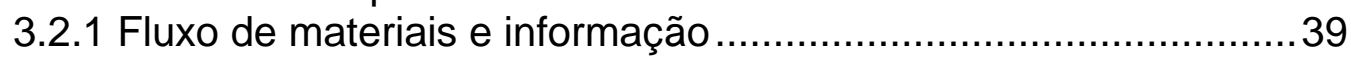

3.3. Visão estratégica da GSK para sua cadeia de suprimentos...........41 4 .

3.4. Processos utilizados na cadeia de suprimentos da GSK ................44 44

3.5. Global Logistics .............................................................. 47

3.6. - Vantagens obtidas pela GSK com a SCM ………................... 49

\section{PLANEJAMENTO DE SUPRIMENTO E GERENCIAMENTO DA} DEMANDA E DO SUPRIMENTO NA CADEIA DA GSK ........................52

4.1. Métodos de planejamento do suprimento ....................................53

4.1.1. Critério de seleção do método de planejamento de suprimento

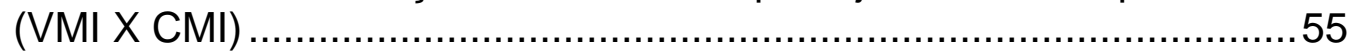

4.2. Gerenciamento da demanda e suprimentos .................................60

4.2.1. Gerenciamento de demanda .................................................66

4.2.2. Gerenciamento do suprimento ...............................................

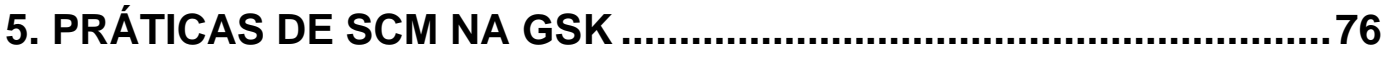

5.1. Distribuição consolidada em parte da Europa .............................. 76

5.2. Redução de inventário através do aumento dos níveis de 
acuracidade de forecast na Finlândia ............................................. 79

5.3. Adoção do CPFR na Suécia .................................................. 81

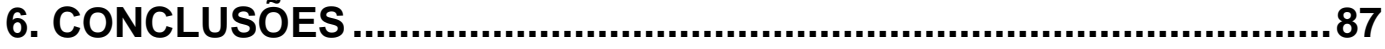

6.1. Recomendações de trabalhos futuros .....................................91

REFERÊNCIAS BIBLIOGRÁFICAS ..................................................93 


\section{Lista de Figuras}

Figura 1-Desenho esquemático do planejamento de necessidade de materiais (MRPI)....

Figura 2-Desenho esquemático do MRPII ..........................................23

Figura 3- S\&OP (PVO) integrado as principais funções da empresa. ...... 25

Figura 4-Abrangência do ERP......................................................... 29

Figura 5-Diagrama do processo de CPFR. .................................... 32

Figura 6-Cinco diferentes cenários de e-business. ............................... 35

Figura 7-Fluxo de informação e produtos na cadeia de suprimentos da

GSK ............................................................................ 40

Figura 8-Processos envolvidos no SCM da GSK ............................. 45

Figura 9-Global Logistics............................................................ 48

Figura 10-Planejamento e gerenciamentos do suprimento e da demanda

na cadeia de suprimentos da GSK ...................................... 52

Figura 11-Matriz decisão CMI/VMI Acuracidade..................................56

Figura 12-Matriz decisão CMI/VMI Nível de serviço............................. 57

Figura 13-Matriz decisão CMI/VMI Lead time .....................................58

Figura 14-Matriz decisão CMI/VMI Back order....................................59

Figura 15-Modelo operacional de demanda e suprimentos. ...................61 61

Figura 16-Sistema integrado de Supply Chain GSK...........................6 63

Figura 17-Tela do Manugistics - Gráfico de planejamento VMI..............65 65

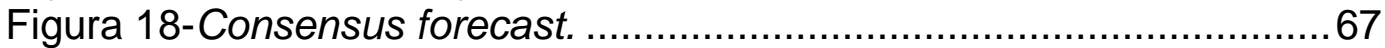

Figura 19-Modelo GSK de Consensus forecast. ..................................69

Figura 20-Revisão de suprimentos fábricas GMS. .............................72 72

Figura 21-Cross dock hub - Europa do leste e central - Antes e depois. 77

Figura 22-Cross dock hub - Europa do leste e central - Antes.................78

Figura 23- Cross dock hub - Europa do leste e central - depois..............78 78

Figura 24-Gráfico aumento de acuracidade forecast GSK Finlândia .......80

Figura 25-Gráfico melhoria nível de estoques GSK Finlândia..................80 80

Figura 26-Cadeia de suprimentos GSK Suécia.................................... 82

Figura 27-Fluxo de informação GSK Suécia - KD .............................. 84

Figura 28-Forecast colaborativo GSK Suécia - KD ............................ 85 


\section{Lista de Tabelas}

Tabela 1-Vantagens e desvantagens do VMI........................................ 33

Tabela 2-Análise do nível de serviço das fábricas. ................................56

Tabela 3-Exemplo de seleção de VMI................................................5

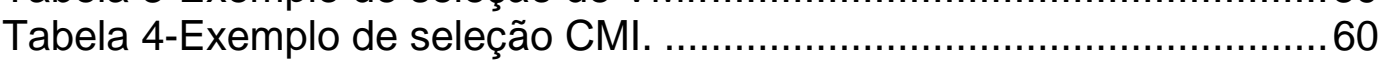

Tabela 5-Lista de Informações recebidas pela GSK de seu distribuidor. . 83 


\section{Lista de abreviações}

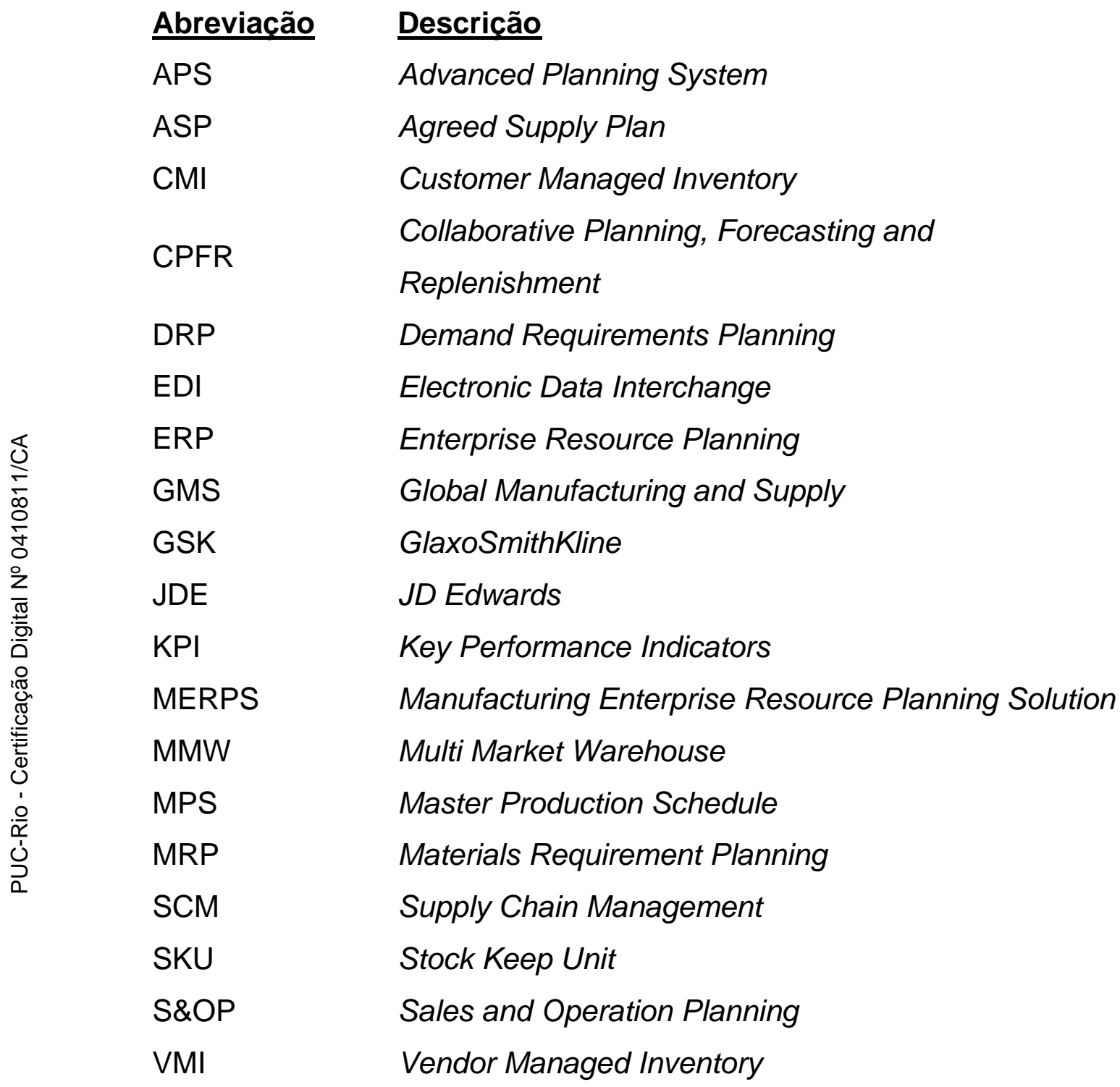

